\title{
Treatment satisfaction significantly improves in patients with multiple sclerosis switching from interferon beta therapy to peginterferon beta-la every 2 weeks
}

This article was published in the following Dove Press journal:

Patient Preference and Adherence

\author{
Barry Hendin' \\ Robert T Naismith ${ }^{2}$ \\ Sibyl E Wray ${ }^{3}$ \\ Deren Huang ${ }^{4}$ \\ Qunming Dong ${ }^{5}$ \\ Terrie Livingston ${ }^{6}$ \\ Daniel L Jones ${ }^{6}$ \\ Crystal Watson ${ }^{7}$ \\ Mehul Jhaveri6
}

'Phoenix Neurological Associates, Phoenix, AZ, USA; ${ }^{2}$ Department of Neurology, Washington University School of Medicine, St Louis, MO, USA; ${ }^{3}$ Hope Neurology MS Center, Knoxville, TN, USA; ${ }^{4}$ Mount Carmel Neuroscience and MDH Research, Westerville, OH, USA; ${ }^{5}$ Biostatistics, Biogen, Cambridge, MA, USA; ${ }^{6}$ US Medical, Biogen, Cambridge, MA, USA; ${ }^{7}$ Global Value and Access, Biogen, Cambridge, MA, USA
Correspondence: Mehul Jhaveri US Medical, Biogen, 225 Binney Street, Cambridge, MA 02।42, USA Email mehul.jhaveri@biogen.com
Objectives: Posthoc analysis of treatment satisfaction in patients switching to subcutaneous (SC) peginterferon beta-1a in the ALLOW study.

Patients and methods: Patients with relapsing multiple sclerosis treated with intramuscular interferon (IFN) beta-1a or SC IFN beta-1a or beta-1b remained on their current therapy for a 4 -week run-in period, followed by a switch to SC peginterferon beta-1a 125 mcg every 2 weeks for 48 weeks. Treatment satisfaction was measured using the Treatment Satisfaction Questionnaire for Medication (TSQM), which covers effectiveness, side effects, convenience, and global satisfaction. Patients completed the TSQM at baseline (prior to starting the 4-week run-in period) and 4, 12, 24, 36, and 48 weeks after switching, and scores were analyzed for the overall population and compared to baseline. Patients reported the severity of flu-like symptoms (FLS) at baseline and with each peginterferon beta-1a injection; clinicians evaluated the occurrence of injection-site reactions (ISRs) after the first dose of peginterferon beta-1a and every 12 weeks thereafter. TSQM scores were stratified by the presence of FLS or ISRs during the study period and by prior IFN therapy use.

Results: For the overall population ( $\mathrm{n}=194)$, convenience and global satisfaction scores significantly improved from baseline at all time points, and side effect satisfaction scores significantly improved up to week 36. Convenience scores significantly improved regardless of FLS, ISRs, or prior IFN therapy. Patients without FLS during the study period showed significant improvements in global satisfaction, but not side effect satisfaction, versus those with FLS. Patients switching from SC IFN therapies achieved greater improvements in treatment satisfaction than patients who switched from intramuscular IFN beta-1a.

Conclusions: Switching relapsing multiple sclerosis patients to SC peginterferon beta-1a from other IFN therapies significantly improved treatment satisfaction and convenience.

Keywords: interferon beta-1a, interferon beta-1b, peginterferon beta-1a, relapsing multiple sclerosis, treatment satisfaction, Treatment Satisfaction Questionnaire for Medication, TSQM, quality of life, patient-reported outcomes

\section{Introduction}

Interferon (IFN) beta-based disease-modifying therapies (DMTs) are well-established treatments approved for relapsing forms of multiple sclerosis (RMS). Intramuscular (IM) IFN beta-1a, subcutaneous (SC) IFN beta-1a, and SC IFN beta-1b have wellcharacterized efficacy and safety profiles. ${ }^{1,2} \mathrm{SC}$ peginterferon beta-1a is the most recently approved IFN for the treatment of RMS, is administered every 2 weeks, and has a safety 
and tolerability profile consistent with the IFN class, with the most common adverse events (AEs) including flu-like symptoms (FLS) and injection-site reactions (ISRs). ${ }^{3-5}$ However, in a Phase I crossover study in healthy volunteers, AEs occurred less frequently with SC peginterferon beta-1a than with $\mathrm{SC}$ IFN beta-1a three times a week over 2 weeks despite $60 \%$ higher levels of drug exposure. ${ }^{3}$ In addition, the ALLOW study, a Phase IIIb, open-label, randomized study in patients with RMS who switched from a nonpegylated IFN to peginterferon beta-1a, found the vast majority of patients $(89.6 \%)$ did not show new or worsening FLS after the switch. ${ }^{6,7}$

While the primary objective of ALLOW was to assess FLS in patients switching to peginterferon beta-1a, ${ }^{6,7}$ participating patients also reported their satisfaction with the therapy. Patient-reported treatment satisfaction is based on factors such as convenience (eg, route of administration and dosing frequency), effectiveness, and side effects. ${ }^{8,9}$ Treatment satisfaction has also been shown to affect treatment adherence and persistence; nonadherence to DMTs has been associated with reduced treatment effectiveness (eg, increased relapse/progression) and may lead to increased hospitalization rates and MS-related costs. ${ }^{10,11}$ These findings highlight the ongoing need to evaluate and improve patients' treatment satisfaction. ${ }^{12}$ Monitoring treatment satisfaction may also help to identify patients who may be at risk of poor medication adherence, ${ }^{13}$ highlighting an opportunity to change medication or implement patient education.

Patient-reported treatment satisfaction has been measured using a variety of instruments, including the Treatment Satisfaction Questionnaire for Medication (TSQM), a validated, psychometrically sound measure for the assessment of patients' satisfaction with medication. ${ }^{13}$ The TSQM has been validated in patients with RMS and has been used to measure satisfaction with DMTs. ${ }^{12,14}$

This posthoc analysis was conducted to assess patientreported treatment satisfaction in patients with RMS who switched from a nonpegylated IFN beta therapy to SC peginterferon beta-1a as a part of the ALLOW study.

\section{Patients and methods}

The ALLOW study design has been described previously. ${ }^{6,7}$ The study enrolled patients aged 18-65 years with RMS treated with a stable dose of nonpegylated IFN for $\geq 4$ months immediately prior to screening. Patients remained on their current therapy for a 4-week run-in period followed by a switch to SC peginterferon beta-1a (125 mcg every 2 weeks) and were followed for a total of 48 weeks.

The primary end point of the ALLOW study was the proportion of patients experiencing new or worsening FLS during the first 8 weeks of SC peginterferon beta-1a therapy. ${ }^{6,7}$ Secondary end points included patient-reported treatment satisfaction.

Patients completed the TSQM at baseline (prior to the run-in period when patients were receiving nonpegylated IFN) and 4, 12, 24, 36, and 48 weeks after switching to peginterferon beta-1a. The TSQM comprises 14 items covering four domains: effectiveness, side effects, convenience, and global satisfaction. ${ }^{13,14}$ The TSQM items utilize a 5- or 7-point Likert-type scale. A score for each domain is calculated by summing the corresponding items transformed on a 0-100 scale; higher values indicate higher satisfaction. ${ }^{15}$

TSQM scores at baseline were compared to TSQM scores at each postswitching visit for the overall efficacy population, defined as patients who received at least one dose of SC peginterferon beta-1a and had TSQM data during the 4-week run-in period and during the postswitching treatment period. All data were analyzed using observed data; no imputation was performed for missing data. TSQM scores were analyzed for the impact of ISRs or FLS occurrence at any point during the study, as well as by prior IFN therapy. FLS were assessed using the total FLS score (FLS-S). Patients were assigned a score from 0 to 3 to their muscle aches, chills, and fatigue as follows: " 0 " for absent; " 1 " for mild, did not interfere with daily activities; " 2 " for moderate, sufficient to interfere with daily activities; and " 3 " for severe, bedrest required. Patients' body temperatures were also assigned a score from 0 to 3 : " 0 " if $<99.1^{\circ} \mathrm{F}$, " 1 " if $\geq 99.1^{\circ} \mathrm{F}$ but $<100.1^{\circ} \mathrm{F}$, " 2 " if $\geq 100.1^{\circ} \mathrm{F}$ but $<101.1^{\circ} \mathrm{F}$, and " 3 " if $\geq 101.1^{\circ} \mathrm{F}$, allowing a total FLS-S ranging from 0 to 12 . As defined in the protocol for the primary end point for the study, a patient with an FLS-S increase of $\geq 2$ from the 4-week run-in period was regarded as having FLS. The effect of prior IFN therapy on changes in baseline TSQM scores was compared in patients previously treated with IM IFN beta-1a or SC IFN beta-1a or beta-1b.

Changes from baseline were recorded using transformed scores between 0 and 100 and analyzed using a paired $t$-test and a significance level of 0.05 . Linear regression analysis was used over all visits. Comparisons between prior IFN therapies, FLS status, and ISR status were analyzed using a two-sample $t$-test and a significance level of 0.05 . To justify the use of $t$-tests, data were also analyzed by Wilcoxon signed-rank test or Wilcoxon rank-sum tests; $P$-values from the two types of tests were similar.

The study was performed in accordance with the Declaration of Helsinki and in compliance with the International Council for Harmonisation and Good Clinical Practice. The study protocol and amendments were approved by each study site's local ethics committee (Table S1), and all patients provided written informed consent. 


\section{Results}

\section{Patients}

Of the 201 patients randomized into ALLOW, 164 (81.6\%) completed the study. Reasons for not completing the study were withdrawal due to AEs (13.4\%), consent withdrawn (3.5\%), and others (1.5\%). The intent-to-treat (ITT) population (the cohort for ALLOW study analysis) comprised 201 randomized patients who received at least one dose of SC peginterferon beta-1a. Baseline characteristics of the ITT population are shown in Table S2. The efficacy population consisted of 194 randomized patients who received at least one dose of SC peginterferon beta-1a and had efficacy data at baseline and postswitching, the cohort for the current analysis. No differences were seen in baseline characteristics between the ITT and efficacy populations.

Nonpegylated IFN therapies taken prior to switching across the ITT population $(\mathrm{n}=201)$ were IM IFN beta-1a (50.8\%), SC IFN beta-1a (25.4\%), SC IFN beta-1b (19.4\%), and unknown $(4.5 \%)$.

\section{TSQM scores: overall efficacy population}

Changes from baseline to each postswitching visit using transformed scores between 0 and 100 are presented for the overall efficacy population in Figure 1. The changes from baseline in convenience and global satisfaction scores were statistically significant at all postswitching time points through week 48 (all $P<0.0001$; Figure 1). Side effect scores were also significantly improved compared with baseline for all visits $(P<0.05)$ except week 48 . Trends toward small improvements in effectiveness scores were seen at later time points, although the changes were not statistically significant.

\section{TSQM scores: by FLS status}

A quarter (49/194) of patients experienced FLS at any point in the study. We analyzed TSQM scores in patients who did versus those who did not experience FLS to determine whether FLS had any impact on treatment satisfaction.

Regardless of whether patients experienced FLS, significant improvements in mean TSQM side effect scores were observed from baseline to week 4 , returning to near-baseline levels by week 48 (Figure 2). Significant improvements in mean TSQM convenience scores from baseline to each subsequent study visit were seen regardless of FLS (all $P<0.0001)$.

Patients who did not experience FLS reported significant improvements from baseline in mean TSQM global satisfaction at all subsequent visits (all $P<0.0001$ ). In contrast, patients who experienced FLS saw an improvement in mean TSQM global satisfaction only from baseline to

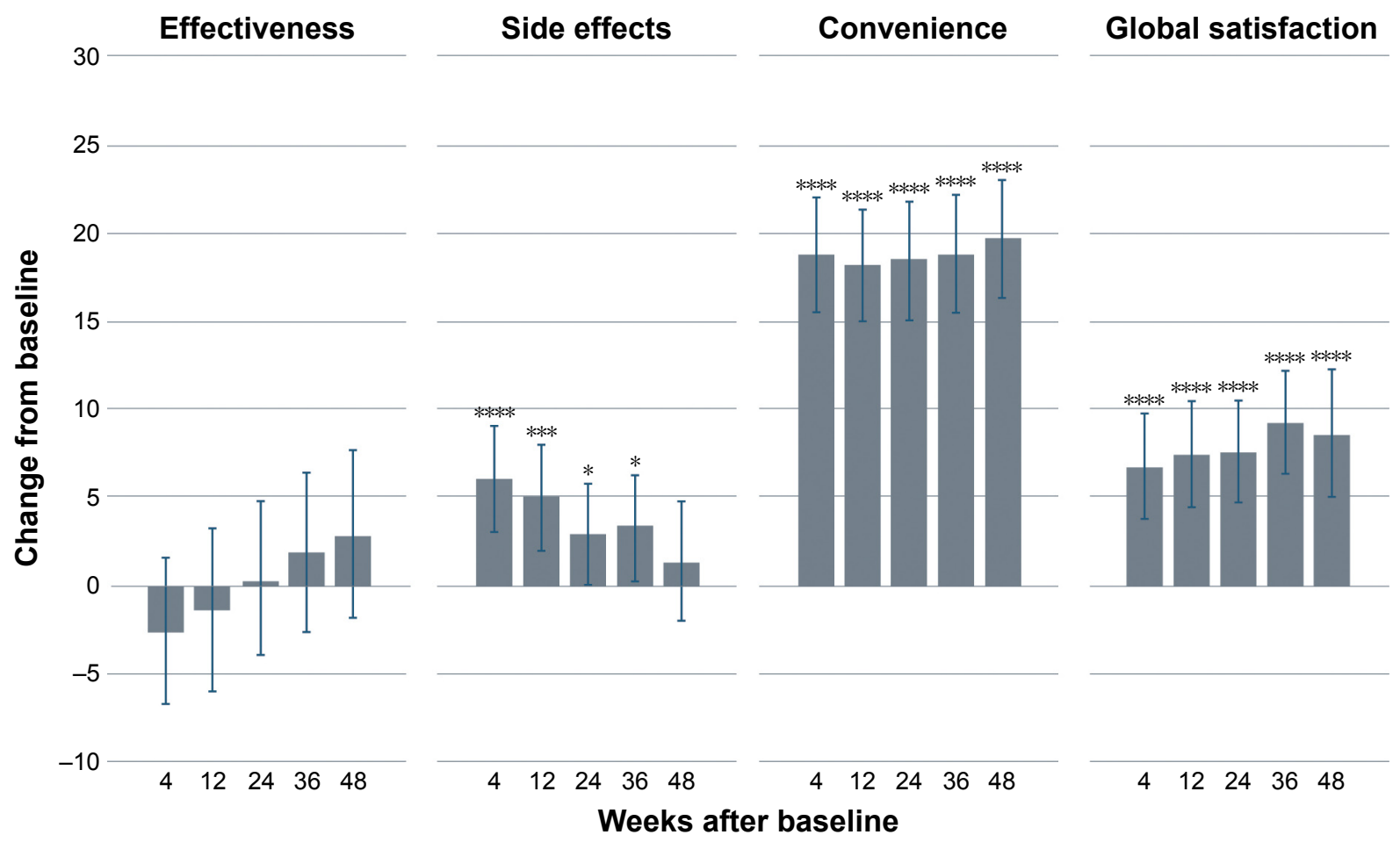

Figure I Improvement in overall TSQM scores from baseline.

Notes: $* P<0.05, * * * P<0.001$, and $* * * * P<0.0001$ compared with baseline. Data are mean values $\pm 95 \% \mathrm{Cl}$.

Abbreviation: TSQM, Treatment Satisfaction Questionnaire for Medication. 


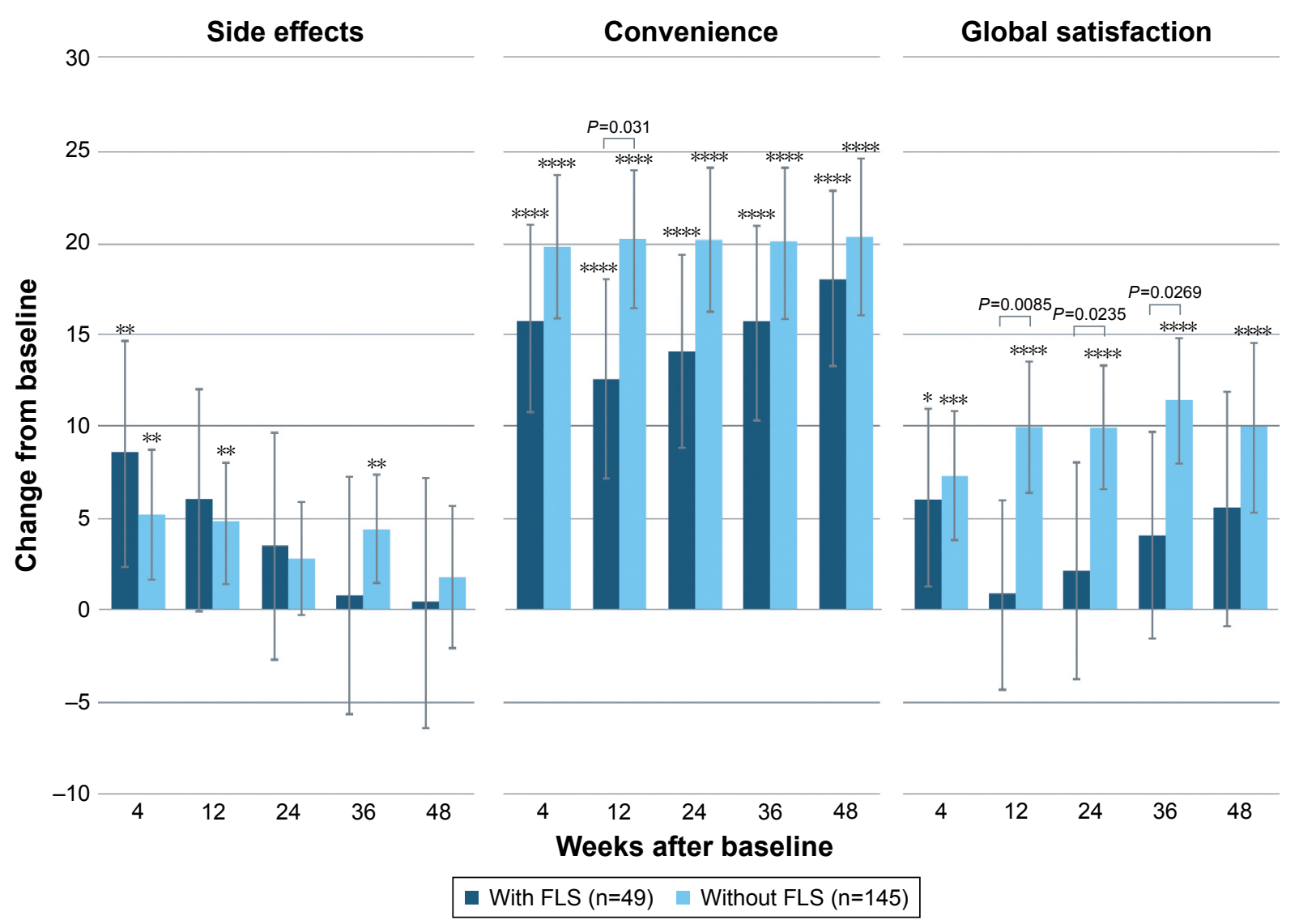

Figure 2 Improvement in TSQM scores (with or without FLS).

Notes: $* P<0.05, * * P<0.01$, $* * * P<0.001$, $* * * * P<0.0001$ compared with baseline. Data are mean values $\pm 95 \% \mathrm{Cl}$. A patient was considered to have FLS if FLS-S total score was $>0$ at any point in the study.

Abbreviations: FLS, flu-like symptoms; FLS-S, flu-like symptoms score; TSQM, Treatment Satisfaction Questionnaire for Medication.

week $4(P<0.05)$. At all other time points, mean global satisfaction scores were numerically higher than baseline but the difference was not statistically significant. Differences in TSQM global satisfaction between the two cohorts, with and without FLS, were statistically significant at weeks 12 , 24, and 36 ( $P<0.05$; Figure 2).

Changes in effectiveness score (not shown) indicated a general improvement for patients both with and without FLS, but none of the changes were significantly different from baseline at any time point.

\section{TSQM scores: by ISR status}

ISRs were experienced by $64.4 \%(125 / 194)$ of patients at any point in the study. TSQM scores were analyzed in patients who did versus those who did not experience ISRs to determine the impact of ISRs on treatment satisfaction.

Statistically significant improvements in mean TSQM side effect scores were observed from baseline to all visits except week 24 for patients who experienced ISRs at any time point in the study (Figure 3). For patients who did not experience ISRs
(69/194), numerical improvements in TSQM side effect scores were observed at weeks $4,12,24$, and 36 , and a numerical reduction was observed at week 48 , but no significant changes from baseline values were observed at any time point.

Regardless of whether patients experienced ISRs, mean TSQM convenience scores were significantly improved from baseline to all subsequent visits (all $P<0.0001$ ). Similarly, whether or not patients experienced ISRs, mean TSQM global satisfaction scores were improved from baseline for all time points except at week 48 .

No significant changes in mean TSQM effectiveness scores from baseline to any subsequent visit for patients with or without ISRs were seen at any point in the study (data not shown).

\section{TSQM scores: by prior IFN}

At screening, 97 patients were receiving IM IFN beta-1a and 89 were receiving SC IFN beta-1a or beta- $1 \mathrm{~b}$. TSQM scores were analyzed by these two prior IFN therapy groups to determine whether prior treatment had any impact on 


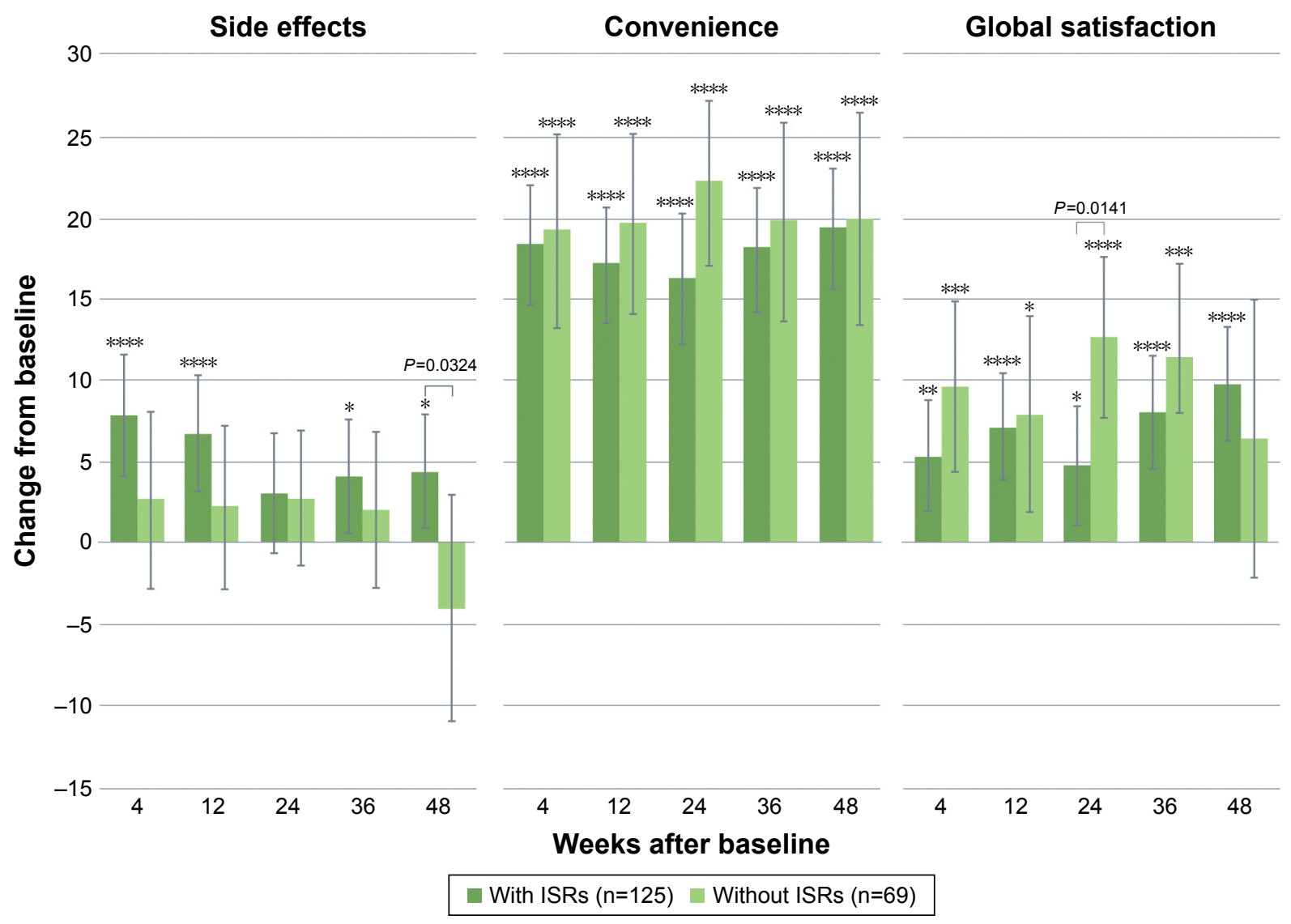

Figure 3 Improvement in TSQM scores (with or without ISRs).

Notes: $* P<0.05$, $* * P<0.0$ I, $* * * P<0.00$ I, $* * * * P<0.000$ I compared with baseline. Data are mean values $\pm 95 \% \mathrm{Cl}$.

Abbreviations: ISR, injection-site reaction; TSQM, Treatment Satisfaction Questionnaire for Medication.

changes in treatment satisfaction associated with the switch to peginterferon beta-1a.

After switching to peginterferon beta-1a, patients reported stable or improved mean TSQM side effect scores from baseline to each visit (Figure 4). Mean TSQM side effect scores improved significantly more for patients transitioning from SC IFN beta-1a or beta- $1 \mathrm{~b}$ than for those transitioning from IM IFN beta- $1 \mathrm{a}$ at weeks 24 and $36(P<0.01)$. In general, for nonpegylated IFN therapies, the largest differences from baseline were seen at the earlier time points, and values returned to baseline or near-baseline by the end of the study.

Mean TSQM convenience scores also significantly improved from baseline to each visit for both nonpegylated IFN groups (all $P<0.0001$ ). The improvement was significantly larger at every visit for the SC IFN beta-1a or beta- $1 \mathrm{~b}$ treatment group compared with the IM IFN beta-1a treatment group.

Significant improvements in mean TSQM global satisfaction scores from baseline were recorded in patients switching from SC IFN beta- $1 \mathrm{a}$ or beta- $1 \mathrm{~b}$ to peginterferon beta- $1 \mathrm{a}$ at all time points, while those previously on IM IFN beta-1a only showed significantly improved global satisfaction at weeks 12, 36, and 48 (Figure 4). Improvements in global satisfaction were significantly greater for the prior SC IFN beta-1a or beta- $1 \mathrm{~b}$ group compared with the IM IFN beta-1a group at weeks 4 and 24, with a trend toward greater improvement at other time points.

Irrespective of prior IFN treatment, TSQM effectiveness scores were generally stable from baseline to each visit after the switch to peginterferon beta-1a (data not shown). Mean values showed a trend in improvement from baseline for patients in the SC IFN beta-1a or beta-1b group, but no changes were significantly different from baseline.

\section{Discussion}

The switch from nonpegylated IFNs to SC peginterferon beta- 1 a every 2 weeks led to significant improvements in patient satisfaction with side effects, convenience, and global satisfaction scores on the TSQM as early as 4 weeks after switching (the earliest time point assessed). Although effectiveness scores were numerically higher (better), no significant changes from baseline were seen, which was 


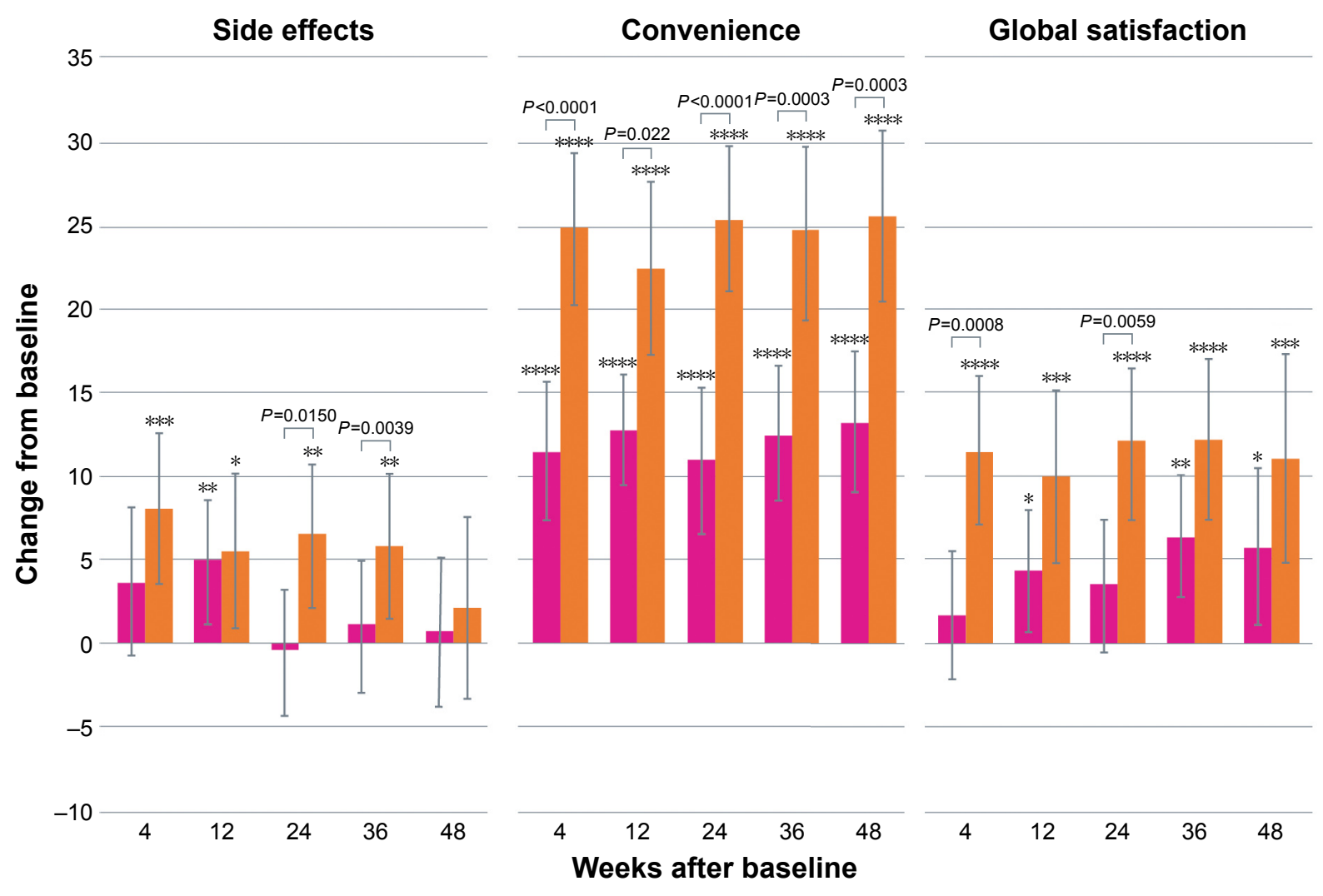

Prior IM IFN beta-1a $(n=97) \quad$ Prior SC IFN beta-1a or beta-1b $(n=89)$

Figure 4 Improvement in TSQM scores (prior IFN treatment).

Notes: $* P<0.05$, $* * P<0.0$ I, $* * * P<0.00$ I, $* * * * P<0.000$ I compared with baseline. Data are mean values $\pm 95 \% \mathrm{Cl}$.

Abbreviations: IFN, interferon; IM, intramuscular; SC, subcutaneous; TSQM, Treatment Satisfaction Questionnaire for Medication.

expected given that the study did not select for patients who were switching due to lack of efficacy.

As FLS are one of the most common AEs associated with IFN therapies, we wanted to determine whether FLS influence treatment satisfaction after a switch to peginterferon beta-1a. Therefore, the data were analyzed by stratifying patients into those with or without FLS during the study. Patients reported improvements from baseline in side effects, convenience, and global satisfaction scores regardless of experiencing FLS. Interestingly, although patients without FLS showed significantly greater improvements in global satisfaction than those with FLS, no significant differences in side effect TSQM scores between the with-FLS and without-FLS treatment groups were seen, suggesting that the improvement in global satisfaction was most likely due to the improvement in convenience scores.

Notably, patients who experienced ISRs showed increased side effect satisfaction scores early after switching to peginterferon beta-1a. One explanation for this seemingly counterintuitive finding may be that these patients may have been experiencing ISRs more frequently with their previous more frequently dosed IFN therapy when compared with every2 -weeks dose administration with peginterferon beta-1a. This observation is consistent with the overall ALLOW study, in which the majority of FLS and ISR events were mild to moderate, with only $2.5 \%$ and $6 \%$ of patients, respectively, discontinuing due to these AEs. ${ }^{6}$

The switch from SC IFN beta-1a or beta-1b to SC peginterferon beta-1a was associated with significantly greater improvements in convenience and global satisfaction than the switch from IM IFN beta-1a. Furthermore, satisfaction with side effects was significantly improved following the treatment switch for patients on prior SC IFN beta-1a or beta-1b, but only significant at one time point for patients on prior IM IFN beta-1a (week 4). This finding may relate back to the baseline incidence of ISRs, which tend to be more common with SC IFN beta-1a or beta-1b than with IM IFN beta- $1 a ;{ }^{16}$ patients on prior SC IFN beta therapies are therefore likely to show greater improvement in satisfaction with side effects. Taken together, these results suggest that SC IFN beta-1a or beta- $1 \mathrm{~b}$ patients are likely to gain the greatest benefit in treatment satisfaction by switching to peginterferon beta-1a. 
This greater treatment satisfaction with peginterferon beta-1a is likely driven largely by differences in dosing schedule between the IFN therapies. In a 2-week period, patients on SC IFN beta-1a or beta-1b inject six or seven times, respectively; those on IM IFN beta-1a inject twice; and those on SC peginterferon beta-1a inject once. It is perhaps not surprising that patients on prior SC IFN beta exhibited greater improvements in treatment satisfaction with peginterferon beta-1a. The reduction in injection frequency likely led to reduced ISR frequency in those who had experienced ISRs on SC IFN. Different routes of administration may also have played a part in differences in convenience scores due to lack of familiarity with SC injections in those who had been treated with IM IFN beta-1a.

Although the TSQM has previously been utilized as a patient-centered end point in RMS, this study is the first to demonstrate that peginterferon beta-1a improves global satisfaction, specifically side effect and convenience scores, in patients with RMS previously treated with nonpegylated IFN therapies. Previous studies have utilized the TSQM to demonstrate improvements in treatment satisfaction over time with other DMTs. ${ }^{17,18}$ One of these studies found a clinically meaningful relationship between each TSQM domain and clinical outcomes such as treatment failure, relapse, and AEs leading to discontinuation. ${ }^{17}$ In another study comparing treatment satisfaction in patients switching from a nonpegylated IFN to fingolimod with those remaining on IFN, significant improvement in all domains of satisfaction was identified. ${ }^{18}$ Two cross-sectional studies demonstrated differences in TSQM scores between DMTs and, in agreement with this study, found differences primarily in side effect and convenience scores. ${ }^{19,20}$

The implications of improved treatment satisfaction are likely to be substantial, potentially leading to increased treatment adherence and, therefore, success. ${ }^{13}$ For example, AEs and perceived lack of effectiveness have been shown to impact long-term persistence with treatment. ${ }^{21,22}$ In addition, improvements in convenience scores on the TSQM have been associated with improved adherence. ${ }^{20}$ Since longer treatment duration has been linked with improved long-term outcomes in patients with RMS, ${ }^{23}$ components of treatment satisfaction that have an impact on persistence and adherence are likely to lead to improved treatment success.

\section{Limitations}

Limitations of this study include the fact that it was not powered to evaluate differences in subgroups, though it did include this as a secondary end point that provides important insights into what is important to patients, and the fact that clinically significant improvement has not been well defined, though previous studies have shown relationships between clinical outcomes and TSQM domains. ${ }^{17}$

\section{Conclusions}

In summary, patients with RMS who switched from nonpegylated IFN beta therapies to SC peginterferon beta-1a reported significant improvements in satisfaction with treatment convenience, side effects, and global satisfaction as early as 4 weeks and continued improvements over 48 weeks. Regardless of FLS or ISR status, treatment with peginterferon beta-1a significantly improved patient-reported satisfaction with the convenience of treatment. Although peginterferon beta-1a improved treatment satisfaction regardless of prior IFN, the greatest improvements in global satisfaction and convenience were seen in patients switching from SC IFN beta-1a or beta-1b. Due to the administration schedule, patients who require a switch may show high levels of satisfaction and also find SC peginterferon beta-1a much more convenient than previous IFNs. Patient perspective on treatment satisfaction is very important to follow in clinical practice when considering IFN therapy.

\section{Acknowledgments}

The authors wish to thank the patients who volunteered for this study and the many site staff members who helped to conduct the study. The authors were assisted in the preparation of the manuscript by Jenna Steere of CircleScience (New York, NY, USA). Writing and editorial support were funded by the study sponsor, Biogen (Cambridge, MA, USA). The authors had full editorial control of the manuscript and provided their final approval for all content.

\section{Author contributions}

All authors contributed toward study design and/or data analysis, as well as drafting and revising the paper, and agree to be accountable for all aspects of the work.

\section{Disclosure}

BH is a paid consultant and/or speaker for Acorda, Biogen, EMD Serono, Genzyme, Mallinckrodt, Novartis, and Teva. RTN is a consultant and/or speaker for Acorda, Alkermes, Biogen, EMD Serono, Genentech, Genzyme, EMD Serono, Novartis, Pfizer, and Questcor. SEW is a paid consultant, speaker, and/or contract researcher for Acorda, Bayer, Biogen, EMD Serono, Genentech/Roche, Genzyme, Novartis, Questcor, Receptos, and Teva. DH is a paid consultant and/or 
speaker for Biogen, Novartis, and Teva Neuroscience. QD and DLJ were employees of Biogen at the time of this work. TL, $\mathrm{CW}$, and MJ are employees and stockholders of Biogen. The authors report no other conflicts of interest in this work.

\section{References}

1. Goodin DS, Frohman EM, Garmany GP Jr, et al; Therapeutics and Technology Assessment Subcommittee of the American Academy of Neurology and the MS Council for Clinical Practice Guidelines. Disease modifying therapies in multiple sclerosis: report of the Therapeutics and Technology Assessment Subcommittee of the American Academy of Neurology and the MS Council for Clinical Practice Guidelines. Neurology. 2002;58(2):169-178.

2. Einarson TR, Bereza BG, Machado M. Comparative effectiveness of interferons in relapsing-remitting multiple sclerosis: a meta-analysis of real-world studies. Curr Med Res Opin. 2017;33(3):579-593.

3. Hu X, Shang S, Nestorov I, et al. COMPARE: pharmacokinetic profiles of subcutaneous peginterferon beta-1a and subcutaneous interferon beta-1a over 2 weeks in healthy subjects. Br J Clin Pharmacol. 2016; 82(2):380-388.

4. Calabresi PA, Kieseier BC, Arnold DL, et al. Pegylated interferon beta-1a for relapsing-remitting multiple sclerosis (ADVANCE): a randomised, phase 3, double-blind study. Lancet Neurol. 2014;13(7):657-665.

5. Kieseier BC, Arnold DL, Balcer LJ, et al. Peginterferon beta-1a in multiple sclerosis: 2-year results from ADVANCE. MultScler. 2015; 21(8):1025-1035.

6. Naismith RT, Hendin B, Wray S, You X, Sabatella G, Zambrano J. ALLOW a phase $3 \mathrm{~b}$ trial characterising flu-like symptoms in patients transitioning to pegylated interferon betala: interim analysis of all patients. Poster presented at: 31 th Congress of the European Committee for Treatment and Research in Multiple Sclerosis; October 7-10, 2015; Barcelona, Spain. P1114.

7. Naismith RT, Hendin B, Wray S, Huang D, You X, Werneburg B. Low risk of new flu-like symptoms in patients transitioning from nonpegylated to pegylated interferon beta-1a and mitigation with scheduled naproxen. Poster presented at: Consortium of Multiple Sclerosis Centers 2016 Annual Meeting; June 1-4, 2016; National Harbor, MD, USA. P-SX12.

8. Brody DS, Miller SM, Lerman CE, Smith DG, Caputo GC. Patient perception of involvement in medical care: relationship to illness attitudes and outcomes. J Gen Intern Med. 1989;4(6):506-511.

9. Barbosa CD, Balp MM, Kulich K, Germain N, Rofail D. A literature review to explore the link between treatment satisfaction and adherence, compliance, and persistence. Patient Prefer Adherence. 2012;6:39-48.

10. Menzin J, Caon C, Nichols C, White LA, Friedman M, Pill MW. Narrative review of the literature on adherence to disease-modifying therapies among patients with multiple sclerosis. J Manag Care Pharm. 2013;19(1 Suppl A):S24-S40.
11. Patti F. Optimizing the benefit of multiple sclerosis therapy: the importance of treatment adherence. Patient Prefer Adherence. 2010;4: $1-9$.

12. Vermersch P, Hobart J, Dive-Pouletty C, Bozzi S, Hass S, Coyle PK. Measuring treatment satisfaction in MS: is the Treatment Satisfaction Questionnaire for Medication fit for purpose? MultScler. 2017;23(4): 604-613.

13. Atkinson MJ, Sinha A, Hass SL, et al. Validation of a general measure of treatment satisfaction, the Treatment Satisfaction Questionnaire for Medication (TSQM), using a national panel study of chronic disease. Health Qual Life Outcomes. 2004;2:12.

14. Ting J, Liu Y, Petrillo J, Giannattasio G, Sabatella G. Treatment satisfaction with disease modifying therapies in multiple sclerosis: a systematic review of studies using the Treatment Satisfaction Questionnaire for Medication (TSQM). Value Health. 2015;18(7):A760-A761.

15. Regnault A, Balp MM, Kulich K, Viala-Danten M. Validation of the Treatment Satisfaction Questionnaire for Medication in patients with cystic fibrosis. J Cyst Fibros. 2012;11(6):494-501.

16. Beer K, Müller M, Hew-Winzeler AM, et al. The prevalence of injectionsite reactions with disease-modifying therapies and their effect on adherence in patients with multiple sclerosis: an observational study. BMC Neurol. 2011;11:144.

17. Vermersch P, Czlonkowska A, Grimaldi LM, et al; TENERE Trial Group. Teriflunomide versus subcutaneous interferon beta-1a in patients with relapsing multiple sclerosis: a randomised, controlled phase 3 trial. Mult Scler. 2014;20(6):705-716.

18. Fox EJ, Edwards K, Burch G, et al; EPOC study investigators. Outcomes of switching directly to oral fingolimod from injectable therapies: results of the randomized, open-label, multicenter, Evaluate Patient OutComes (EPOC) study in relapsing multiple sclerosis. MultScler Relat Disord. 2014;3(5):607-619.

19. Haase R, Kullmann JS, Ziemssen T. Therapy satisfaction and adherence in patients with relapsing-remitting multiple sclerosis: the THEPA-MS survey. Ther Adv Neurol Disord. 2016;9(4):250-263.

20. Glanz BI, Musallam A, Rintell DJ, Chitnis T, Weiner HL, Healy BC. Treatment satisfaction in multiple sclerosis. Int J MS Care. 2014;16(2): 68-75.

21. Ross AP. Tolerability, adherence, and patient outcomes. Neurology. 2008;71(24 Suppl 3):S21-S23.

22. Visser LH, van der Zande A. Reasons patients give to use or not to use immunomodulating agents for multiple sclerosis. Eur J Neurol. 2011;18(11):1343-1349.

23. Kappos L, Kuhle J, Multanen J, et al. Factors influencing long-term outcomes in relapsing-remitting multiple sclerosis: PRISMS-15. J Neurol Neurosurg Psychiatry. 2015;86(11):1202-1207. 


\section{Supplementary materials}

Table SI List of local ethics committees

\begin{tabular}{lc}
\hline Name & Location \\
\hline Copernicus Group Independent Review Board & Durham, NC, USA \\
Western IRB & Puyallup, WA, USA \\
St Elizabeth's Medical Center IRB & Boston, MA, USA \\
Washington University Human Research Protection Office & St Louis, MO, USA \\
Lahey Hospital \& Medical Center IRB & Burlington, MA, USA \\
The University of Kansas Medical Center IRB & Kansas City, KS, USA \\
Christiana Care Institutional Review Board & Newark, DE, USA \\
University of Massachusetts Medical School IRB & Worcester, MA, USA \\
Oklahoma Medical Research Foundation IRB & Oklahoma City, OK, USA \\
Henry Ford Health System's IRB & Detroit, MI, USA \\
Providence Health \& Services IRB & Portland, OR, USA \\
\hline
\end{tabular}

Abbreviation: IRB, institutional review board.

Table S2 Baseline characteristics of patients enrolled in the ALLOW study

\begin{tabular}{lc}
\hline Characteristics & $\begin{array}{l}\text { ITT population } \\
\text { (N=20I) }\end{array}$ \\
\hline Age, years & $49.8(9.9)$ \\
$\quad$ Mean (SD) & $5 I(I 8-65)$ \\
$\quad$ Median (range) & $163(8 I .1)$ \\
Female, $n$ (\%) & \\
Time since MS symptom onset, years & $13.4(8.5)$ \\
$\quad$ Mean (SD) & $12(I-52)$ \\
$\quad$ Median (range) & $5.4(4.62)$ \\
Time since most recent relapse, mean (SD), years & \\
Number of relapses within the last year & $0.2(0.63)$ \\
$\quad$ Mean (SD) & $0(0-4)$ \\
$\quad$ Median (range) & \\
\hline
\end{tabular}

Abbreviations: ITT, intent-to-treat; MS, multiple sclerosis.

\section{Publish your work in this journal}

Patient Preference and Adherence is an international, peer-reviewed, open access journal that focuses on the growing importance of patient preference and adherence throughout the therapeutic continuum. Patient satisfaction, acceptability, quality of life, compliance, persistence and their role in developing new therapeutic modalities and compounds to optimize clinical outcomes for existing disease states are major areas of interest for the journal. This journal has been accepted for indexing on PubMed Central. The manuscript management system is completely online and includes a very quick and fair peer-review system, which is all easy to use. Visit http://www. dovepress.com/testimonials.php to read real quotes from published authors.

\footnotetext{
Submit your manuscript here: http://www.dovepress.com/patient-preference-and-adherence-journal
} 